\title{
Stereodynamics and Control Effects in the Ultraviolet Photolysis of $\mathrm{Ar}-\mathrm{HBr}$
}

\author{
R. Prosmiti and A. García-Vela* \\ Instituto de Matemáticas y Física Fundamental, \\ C.S.I.C., Serrano 123, 28006 Madrid, Spain
}

\begin{abstract}
The ultraviolet photolysis of the $A r-H B r$ cluster is studied by means of quasiclassical trajectory simulations. The goal is to investigate the effect of the initial distribution of intracluster orientations on the photolysis process. Cluster photolysis is simulated starting from two different initial states of $\mathrm{Ar}-$ $\operatorname{HBr}(v=1)$, namely the ground and the first excited van der Waals (vdW) states corresponding to the $\mathrm{Ar}-\mathrm{H}-\mathrm{Br}$ and $\mathrm{Ar}-\mathrm{Br}-\mathrm{H}$ isomers, respectively. These two states represent different initial situations for hydrogen dissociation, which in the first case is hindered to a large extent, and in the second case is not. The probability for the fragmentation pathway into $H$ and $A r-$ $\mathrm{Br}$ radical fragments is found to be substantially higher (and therefore the efficiency of the total fragmentation pathway, into $\mathrm{H}+\mathrm{Ar}+\mathrm{Br}$, is lower) for the cluster initial state where $H$ dissociation is less hindered. The effect of the angular distributions shape of the initial state manifests clearly also in the state distributions of the different photofragments. It is concluded that selecting the initial state of the cluster on the basis of the shape of its angular distribution provides an efficient means to control the yield of the different fragmentation pathways, and the final states of their corresponding photolysis products.
\end{abstract}

*E-mail: garciavela@imaff.cfmac.csic.es

Running title: Ultraviolet Photolysis of $\mathrm{Ar}-\mathrm{HBr}$ 


\section{INTRODUCTION}

The ultraviolet (UV) photodissociation of weakly bound hydrogen-bonded clusters has attracted a growing attention in the last decade. Several experimental groups have investigated the UV photolysis of small clusters such as $\mathrm{Ar}-\mathrm{HBr}{ }^{1,2}$ $\mathrm{Ar}-\mathrm{HI},{ }^{2}(\mathrm{HI})_{2},{ }^{3}(\mathrm{HCl})_{2},{ }^{4,5} \mathrm{Ar}-\mathrm{H}_{2} \mathrm{O},{ }^{6,7} \mathrm{Ar}_{n}-\mathrm{H}_{2} \mathrm{~S}(n \leq 2),{ }^{8}$ and larger clusters like $(\mathrm{HI})_{n},{ }^{9}$ and $(\mathrm{HBr})_{n}{ }^{10}$ with $n>2$. By UV photoexcitation to a repulsive electronic state of the chromophore ( $\mathrm{HX}$ or $\mathrm{H}_{2} Y, \mathrm{X}=$ halogen, $\mathrm{Y}=\mathrm{O}, \mathrm{S}$ ), a large amount of energy is deposited, causing fast ejection of a hot $H$ fragment. Upon the $H$ atom recoil, cluster fragmentation can follow different pathways. Direct dissociation of the hydrogen may leave behind open-shell radical complexes ${ }^{2-4,8}$ with a distribution of rovibrational excitations, which allows one to probe the open-shell interactions involved. When the recoiling hydrogen collides with the species weakly bound to the chromophore, more extensive fragmentation of the cluster can occur.

A number of theoretical simulations of the UV photolysis of different H-bonded clusters has been performed. Among the systems studied are $X e_{n}-H I(n=$ $1-12),{ }^{11} \mathrm{Ar}-\mathrm{HCl},{ }^{12-19} \mathrm{Ar}{ }_{n}-\mathrm{HCl}(\mathrm{n} \geq 2),{ }^{20,22}(\mathrm{HCl}){ }_{2},{ }^{23} \mathrm{Ar}-\mathrm{HBr},{ }^{1,24} \mathrm{Ar}{ }_{n}-\mathrm{HBr}$ $(n \geq 2),{ }^{25} \mathrm{Ar}_{n}-\mathrm{HF}(n=1-14,54),{ }^{26,27} \mathrm{Ar}-\mathrm{H}_{2} \mathrm{O},{ }^{28}$ and $A r_{n}-\mathrm{H}_{2} \mathrm{~S}(n \leq 2) .{ }^{29}$ Out of the above systems, the $\mathrm{Ar}-\mathrm{HCl}$ cluster has been the most extensively studied. This is the first system for which it has been predicted theoretically ${ }^{16,18,19}$ that, in addition to cluster dissociation into three fragments, UV photolysis can occur through another fragmentation pathway producing $\mathrm{Ar}-\mathrm{Cl}$ radical fragments. This is in accordance with the experimental evidence found for other clusters like $\mathrm{Ar}-\mathrm{HI},{ }^{2}(\mathrm{HCl})_{2},{ }^{4}$ and $\mathrm{Ar}{ }_{n}-\mathrm{H}_{2} \mathrm{~S}(n \leq 2){ }^{8}$ A global picture of the $\mathrm{Ar}-\mathrm{HCl} \mathrm{UV}$ photolysis process has been recently suggested, ${ }^{19}$ which could also be valid for other hydrogen-bonded clusters. In this picture the probability of each fragmentation pathway (total or partial cluster fragmentation) would be greatly determined by a competition where interference effects between the two pathways appear to play a major role. One of the conclusions of the works on $\mathrm{Ar}-\mathrm{HCl}$ was that this competition between fragmentation pathways is strongly dependent on the initial 
distribution of angular intracluster geometries excited. ${ }^{16,18,19}$ In the case of $A r-$ $\mathrm{HCl}$, dissociation into three fragments involves at least one collision of the recoiling hydrogen with the $A r$ atom. This situation occurs when the $H$ recoil is hindered by the $A r$ obstacle, for initial angular geometries relatively close to the collinear equilibrium one. By contrast, for initial angles far from the collinear equilibrium geometry, for which the $H$ can recoil freely, the probability of partial fragmentation of the cluster producing $\mathrm{Ar}-\mathrm{Cl}$ radicals is enhanced. Thus, the weight of the initial angular geometries, determined by the initial state of the cluster prepared by UV pumping, governs to a great extent the branching ratio between the different fragmentation pathways and their products.

The influence of the initial distribution of intracluster angular geometries has also been found in the UV photolysis of $A r_{n}-H C l,{ }^{22} A r_{n}-H B r,{ }^{25(b)}$ and $A r_{12}-H F^{27}$ clusters. In these works it has been shown that by exciting the bending mode of the $H X$ subunit, prior to the UV pumping, the temporary trapping of the dissociating hydrogen within the cluster (also called cage effect) can be efficiently "turned off". Due to the hydrogen-bonding interactions in this type of clusters, their equilibrium geometry in the ground state is such that hydrogen recoil is hindered by the solvent cage. The $H X$ bending excitation has the effect of changing this situation, preparing an initial state of the cluster with an angular distribution where the $H$ recoil is less (or even not) hindered by the solvent cage. A similar effect was found in the UV photolysis of the $\mathrm{Cl}-\mathrm{HCl}$ system, ${ }^{30}$ where bending excitation of the cluster prior to the UV excitation increased the probability of the $\mathrm{Cl}_{2}$ product by a factor of 2 . This case presents qualitative similarities with the formation of $\mathrm{Ar}-\mathrm{Cl}$ radicals after UV photodissociation of $\mathrm{Ar}-\mathrm{HCl}$. It should be noted that excitation of the hydrogen bending motion is not always required in order to prepare an initial distribution of angular geometries from which the hydrogen can recoil freely. In this sense, it has been predicted ${ }^{2,31,32}$ that, for the $A r H I$ cluster, the equilibrium geometry in the ground state corresponds to the isomer $A r-I-H$, with the hydrogen pointing outwards the $A r \cdots I$ cage. 
Thus, selecting an initial cluster state with the proper shape of the distribution of intracluster angular geometries, before UV excitation, appears as an efficient way to control the yield of specific product fragments of the cluster photolysis. The aim of the present work is to explore this possibility in the case of the UV photodissociation of the $\mathrm{Ar}-\mathrm{HBr}$ cluster. The potential-energy surface of Han et al. ${ }^{33}$ for the ground electronic state of $A r-H B r$ predicts that for the $v=1$ vibrational state of $H B r$, the ground vdW state corresponds to the $A r-H-B r$ geometry, while the first excited vdW state is associated with the $A r-B r-H$ geometry. Therefore two states are identified in $A r-H B r$ with very different shapes (actually opposite) of the angular distribution. This makes this system a very suitable candidate to investigate the effect of the initial angular geometry distribution in the photolysis process. For this purpose, the UV photodissociation of $A r-H B r$ has been simulated, by means of quasiclassical trajectories, starting from both the ground and the first excited vdW states of the cluster. Simulations at different excitation energies of $\mathrm{Ar}-\mathrm{HBr}$ in the upper electronic state were carried out. The main focus of the analysis is on how the different shape of the initial angular distribution affects the branching ratio between the partial and total fragmentation pathways of the cluster, and the state distributions of the corresponding product fragments.

The organization of the paper is the following. In Sec. II the potential-energy surfaces and the quasiclassical methodology used are described. Results are presented and discussed in Sec. III. Concluding remarks are given in Sec. IV.

\section{THEORETICAL TREATMENT}

\section{A. Potential-energy surfaces}

Photolysis of $\mathrm{Ar}-\mathrm{HBr}$ occurs upon UV excitation of $\mathrm{HBr}$ from its ground electronic state $X^{1} \Sigma^{+}$to the repulsive excited state $A^{1} \Pi$ [which correlates adiabatically with the $H\left({ }^{2} S\right)+\operatorname{Br}\left({ }^{2} P_{3 / 2}\right)$ asymptote]. Such an excitation is simulated by promoting vertically the initial state of the cluster, calculated in the $X^{1} \Sigma^{+}$potential 
surface, to the excited $A^{1} \Pi$ surface, assuming a Franck-Condon transition.

The ground-state potential surface is modelled as a sum of an atom-atom potential for the $\mathrm{H}-\mathrm{Br}$ interaction plus a term reproducing the vdW interaction,

$$
V_{g}(r, R, \theta)=V_{H-B r}^{g}(r)+V_{v d W}(R, \theta),
$$

with $(r, R, \theta)$ being Jacobian coordinates where $r$ is the $H-B r$ distance, $R$ is the separation from the $A r$ atom to the center-of-mass of $\mathrm{HBr}$, and $\theta$ is the angle between the vectors $\mathbf{r}$ and $\mathbf{R}$. In the definition adopted here $\theta=0$ corresponds to the collinear configuration where the hydrogen points towards the $A r$ atom. For the $V_{H-B r}^{g}$ term a Morse function is used with parameters $D=3.92 \mathrm{eV}, r_{e}=1.414$ $\AA$, and $\alpha=1.821 \AA^{-1}{ }^{34}$ The potential for the vdW interaction is taken to be the intermolecular $A r-\operatorname{HBr}(v=1)$ potential of Han et al. ${ }^{33}$ which was fitted using data of infrared spectra. The functional form of this intermolecular potential is very similar to the $H 4$ potential of Hutson ${ }^{31}$ for $A r-H B r(v=0)$.

The excited electronic state potential is represented as a sum of pairwise interactions between the atoms of the system

$$
V_{e}=V_{H-B r}^{e}+V_{H-A r}^{e}+V_{A r-B r}^{e}
$$

The $H-B r$ interaction in the $A^{1} \Pi$ excited state, $V_{H-B r}^{e}$, is represented by an exponential function

$$
V_{H-B r}^{e}(r)=A e^{-\beta r},
$$

with $A=61.2 \mathrm{eV}$ and $\beta=2.01 \AA^{-1} \cdot{ }^{35,36}$ Empirical potentials have been used for the $H-A r^{37}$ and $A r-B r^{38}$ (in the $X 1 / 2$ ground electronic state) interactions.

\section{B. Dynamics simulations}

The initial state of $A r-H B r(v=1)$ in the $X^{1} \Sigma^{+}$surface is expressed as

$$
\Phi(r, R, \theta)=\chi_{v=1}(r) \psi(R, \theta)
$$

where $\chi_{v=1}(r)$ is the $v=1$ vibrational eigenstate of the $V_{H-B r}^{g}(r)$ potential. The function $\psi(R, \theta)$ describing the vdW modes is calculated variationally by representing the vibrationally-averaged Hamiltonian $\hat{H}_{v, v}=<\chi_{v=1}(r)|\hat{H}|_{\chi_{v=1}}(r)>$ (being 
$\hat{H}$ the Hamiltonian of the system) on a suitable basis set and diagonalizing. The energies obtained for the ground and first excited vdW states are $-121.61 \mathrm{~cm}^{-1}$ and $-112.47 \mathrm{~cm}^{-1}$, respectively, relative to the $v=1$ energy level of $\mathrm{HBr}$.

The quantum distributions associated with the calculated ground and first excited vdW states of $A r-H B r(v=1), \Phi(r, R, \theta)$, are used to sample initial conditions corresponding to different excitation energies of the cluster in the upper electronic surface. The excitation energies investigated cover the range of the cluster absorption spectrum. Starting from these initial conditions the cluster photodissociation is simulated by running classical trajectories in the $A^{1} \Pi$ excited surface. Zero total angular momentum of the system is assumed.

In order to sample the initial conditions for the trajectories, a sampling method previously suggested ${ }^{39}$ and applied to study the UV photolysis of $A r-H C l^{19}$ has been used. In this method a quantum initial phase-space distribution is defined as $\left|\Phi(r, R, \theta) \tilde{\Phi}\left(p_{r}, p_{R}, l\right)\right|^{2} \sin \theta$, where $\tilde{\Phi}\left(p_{r}, p_{R}, l\right)$ is the momentum-space representation of $\Phi(r, R, \theta)$, and $l$ is the rotational state associated with the angular mode $\theta$. A phase-space grid is then defined and initial conditions are sampled uniformly within that grid following the above distribution. We note that this phase-space distribution retains the quantum character and shape of the cluster initial state. This feature of the sampling method is very important, since the goal of the present work is to elucidate the effect of the shape of the initial intracluster angular geometry distribution in the photodissociation dynamics.

The energy dispersion of the initial conditions sampled for each excitation energy $E$ of the parent cluster in the upper potential surface is required to be $\Delta E=$ $\pm 10 \mathrm{~cm}^{-1}$. This ensures that the calculated product state distributions are energyresolved. The limit $E=0$ corresponds to three separated atoms. Calculations were carried out for the cluster excitation energies $E=1.83 \mathrm{eV}, 2.40 \mathrm{eV}, 2.97 \mathrm{eV}, 3.54 \mathrm{eV}$, $4.11 \mathrm{eV}, 4.68 \mathrm{eV}, 5.25 \mathrm{eV}$, and $5.82 \mathrm{eV}$, and the number of trajectories integrated was 3155, 50066, 66723, 15253, 24884, 30947, 21283, and 4786, respectively, for the ground vdW state of $A r-H B r(v=1)$, and 3055, 49450, 65258, 13929, 27155, 
31210, 22951, and 4570, respectively, for the first excited vdW state. The number of initial conditions associated with a specific excitation energy is related to the probability given by the corresponding phase-space distribution for that energy. The trajectories were integrated up to a final time $t_{f}=200 \mathrm{fs}$ with a time step $\Delta t=0.05 \mathrm{fs}$. This integration time is enough taking into account that the cluster photodissociation dynamics is essentially over for a time $t<100 \mathrm{fs}$. At $t_{f}=200 \mathrm{fs}$ the $\mathrm{H}-\mathrm{Br}$ distance is larger than 20a.u. for all trajectories integrated.

At this point a comment is due on the limitations of the present description of the photolysis process. We are assuming that the photodissociation dynamics occurs on a single excited potential surface, that of the $A^{1} \Pi$ electronic state. Effects derived from nonadiabatic couplings to other electronic states are not taken into account in the dynamical treatment. In addition, the classical dynamics approach employed is approximate, and this may have an effect considering the presence of a typically quantum particle like the $H$ atom. Possible quantum effects are therefore missing in the dynamics. The present work aims at predicting qualitative trends and effects, and in this sense, the treatment applied here represents a compromise between efficiency of the calculations and reliability of the predictions. We believe that the present level of description is sufficient to capture the main dynamical trends. This is supported by the agreement found earlier in a comparison between experiment and classical simulations of the UV photolysis of $A r-H B r(v=0) .{ }^{1}$

It is also interesting to comment on the connection of the present calculations with a possible experiment. Such an experiment would involve a two-photon excitation of the cluster. The cluster would be first excited by an infrared photon from the $A r-H B r(v=0)$ ground state to either the ground or the first excited vdW state of $A r-H B r(v=1)$, in the lower electronic surface. Then a second UV photon would pump the system to the upper electronic surface where photolysis takes place. This type of double-resonance excitation experiment has been carried out earlier with other related hydrogen-bonded clusters like $(\mathrm{HCl})_{2},{ }^{4}$ and $\mathrm{Ar}-\mathrm{H}_{2} \mathrm{O} .{ }^{6}$

\section{RESULTS AND DISCUSSION}




\section{A. Initial angular distributions}

The distributions of initial angular geometries associated with the ground and first excited vdW states of $A r-H B r(v=1)$,

$$
P(\theta)=\int d R|\psi(R, \theta)|^{2} \sin \theta
$$

are shown in Fig. 1. The angular distribution corresponding to the ground vdW state of $\mathrm{Ar}-\mathrm{HCl}(v=0)^{16,18,19}$ is also displayed in the figure for the sake of comparison. The two angular distributions of $A r-H B r(v=1)$ are nearly mirror images with respect to $\theta \simeq 100^{\circ}$. Both distributions spread over the whole angular range, although most of their probability concentrates at angles closer to the $\mathrm{Ar}-\mathrm{H}-\mathrm{Br}$ geometry (ground vdW state), or to the $A r-B r-H$ geometry (excited vdW state). It should be noted, however, that each distribution displays a remarkable intensity (higher in the case of the excited vdW state) at opposite geometries to those of maximum probability. This makes a difference between the initial angular distributions of the ground vdW states of $A r-H B r(v=1)$ and $A r-H C l(v=0)$, although these two distributions are qualitatively similar.

\section{B. Probability of $A r-B r$ formation}

Photolysis of $\mathrm{Ar}-\mathrm{HBr}$ can occur through two possible fragmentation pathways,

$$
\begin{aligned}
& A r-H B r+\hbar \omega \rightarrow H+A r-B r \\
& A r-H B r+\hbar \omega \rightarrow H+A r+B r
\end{aligned}
$$

which will be termed partial fragmentation (PF) pathway, Eq. (6), and total fragmentation (TF) pathway, Eq. (7). Cluster fragmentation via these two pathways was investigated for several excitation energies of the system in the range of the absorption spectrum, starting from both the ground and the first excited vdW state.

One of the most interesting aspects of the photolysis process is how the probability of the PF and TF pathways behaves for different excitation energies, when two 
different initial angular distributions of the cluster, like those of Fig. 1, are prepared upstairs. This has been explored by calculating the probability of the PF pathway, or equivalently, the probability of formation of $A r-B r$ radical complexes, $P_{A r-B r}$. By solving numerically the equation

$$
\left[-\frac{\hbar^{2}}{2 \mu_{A r-B r}} \frac{\partial^{2}}{\partial r^{\prime 2}}+\frac{j(j+1) \hbar^{2}}{2 \mu_{A r-B r}\left(r^{\prime}\right)^{2}}+V_{A r-B r}^{e}\left(r^{\prime}\right)\right] \varphi_{\nu}^{(j)}\left(r^{\prime}\right)=E_{\nu, j} \varphi_{\nu}^{(j)}\left(r^{\prime}\right),
$$

one finds that the potential $V_{j}\left(r^{\prime}\right)=V_{A r-B r}^{e}\left(r^{\prime}\right)+j(j+1) \hbar^{2} / 2 \mu_{A r-B r}\left(r^{\prime}\right)^{2}$ supports bound and/or quasibound (due to the rotational barriers) states of $A r-B r$ up to a maximum $j$ value $j_{\max }=74$. In Eq. (8) $r^{\prime}$ is the $A r-B r$ distance, and $V_{A r-B r}^{e}$ is the $A r-B r$ interaction potential in the upper electronic surface [see Eq. (2)].

The $\mathrm{Ar}-\mathrm{Br}$ formation is checked for each trajectory at final time in the following way. The coordinates $r, R, \theta$ and conjugate momenta $p_{r}, p_{R}, p_{\theta}$ used to integrate the trajectories are transformed to the new coordinates $r^{\prime}, R^{\prime}, \theta^{\prime}$ and momenta $p_{r^{\prime}}, p_{R^{\prime}}, p_{\theta^{\prime}}$, where $R^{\prime}$ is the distance between $H$ and the center-of-mass of $A r-B r$, and $\theta^{\prime}$ is the angle between the vectors $\mathbf{R}^{\prime}$ and $\mathbf{r}^{\prime}$. In this new set of Jacobian coordinates the energy of the $A r-B r$ subunit, $E_{A r-B r}^{\text {class }}=p_{R^{\prime}}^{2} / 2 \mu_{A r-B r}+$ $p_{\theta^{\prime}}^{2} / 2 \mu_{A r-B r}\left(r^{\prime}\right)^{2}+V_{A r-B r}^{e}\left(r^{\prime}\right)$ is calculated, and the $j$ state is identified by solving the equation $p_{\theta^{\prime}}^{2}=j(j+1)$. A trajectory is considered to produce an $A r-B r$ radical fragment if the two following criteria are simultaneously fulfilled. These criteria are that $E_{A r-B r}^{\text {class }} \leq E_{\nu, j}^{\max }$, where $E_{\nu, j}^{\max }$ is the highest (bound or quasibound) energy level supported by the potential $V_{j}\left(r^{\prime}\right)$, and that the $A r-B r$ distance is smaller than the distance corresponding to the top of the $j(j+1) \hbar^{2} / 2 \mu_{A r-B r}\left(r^{\prime}\right)^{2}$ barrier. The number of trajectories leading to $A r-B r$ products, $P_{A r-B r}(E)$, gives the probability of the PF pathway for the excitation energy $E$. Dividing this number by the total number of trajectories integrated for each energy, $P_{A r-B r}(E) / P_{\text {total }}(E)$, provides the percentage of the probability of the PF pathway in the photolysis process. The percentage of the TF pathway is obtained as $P_{T F}(E) / P_{\text {total }}(E)=1-P_{A r-B r}(E) / P_{\text {total }}(E)$.

The behavior of the ratio $P_{A r-B r} / P_{t o t a l}$ is shown in Fig. 2 for different energies $E$ of the cluster when it is prepared upstairs from both the ground and the first excited vdW states. The ratio $P_{A r-C l} / P_{\text {total }}$ calculated classically in a previous work ${ }^{19}$ for 
the ground vdW state of $A r-H C l(v=0)$ is also shown in Fig. 2 for the sake of comparison. Two interesting features are observed in the curves of Fig. 2. One of them is the high yield of $A r-B r$ products found over the whole range of excitation energies for the two initial states of the cluster. This yield is particularly remarkable for the ground vdW state of $A r-H B r(v=1)$ when compared to the $A r-C l$ yield obtained for $\mathrm{Ar}-\mathrm{HCl}(v=0)$, taking into account that the initial distributions of hydrogen orientations prepared in both cases are similar, with the hydrogen largely blocked by the $A r$ atom. The other feature is that the yield of $A r-B r$ products is substantially higher when $A r-\operatorname{HBr}(v=1)$ is pumped upstairs from the first excited vdW state, indicating that there is indeed an effect produced by the shape of the initial angular distribution of the cluster.

As the excitation energy of the parent cluster increases the energy available for the weakly bound radical fragment increases as well, causing a decrease of its survival probability. In this line Nesbitt and co-workers proposed a "gentle recoil" mechanism to explain the formation of radicals found after UV photolysis of $A r_{n}-H_{2} S(n \leq 2) .{ }^{8}$ Following this direct dissociation mechanism, the $H$ would recoil carrying most of the excess energy of the cluster, due to its light mass. Thus, the probability of radical formation would be essentially determined by the amount of energy available for the radical and the extent to which the radical can accommodate that energy without breaking. The gentle recoil mechanism is expected to operate in the UV photolysis of both $A r-H C l$ and $A r-H B r$. In addition, side collisions of the recoiling hydrogen with the $A r$ atom, the strength of which is determined by the initial orientation of the hydrogen, transfer energy to the radical bond and they also contribute to diminish the radical survival probability. The initial distributions of hydrogen orientations of the ground vdW states of $A r-\operatorname{HBr}(v=1)$ and $A r-$ $\operatorname{HCl}(v=0)$ are qualitatively similar (see Fig. 1), and large differences are not expected between the two excited electronic potential surfaces (in fact, the $H-A r$ interaction is the same for both systems). Therefore, in principle one would expect a more similar behavior of the yields of $\mathrm{Ar}-\mathrm{Br}$ and $\mathrm{Ar}-\mathrm{Cl}$ radicals when the 
ground vdW states of the corresponding parent clusters are excited. However, the yield of $A r-B r$ produced from the $A r-H B r(v=1)$ ground vdW state decreases much more slowly with increasing $E$.

The high yield of $A r-B r$ radicals for the ground vdW state of the cluster can be explained by the combination of two factors. One factor is that for a given excitation energy, the hydrogen gets a larger share of energy upon photodissociation from $\mathrm{HBr}$ than from $\mathrm{HCl}$, therefore leaving an amount of energy available for $\mathrm{Ar}-\mathrm{Br}$ smaller than that for $\mathrm{Ar}-\mathrm{Cl}$. The effect of an increasing radical survival with increasing radical mass was already anticipated. ${ }^{18}$ In addition, the lowest bound and highest quasibound energy levels of $A r-B r$ are $117.6 \mathrm{~cm}^{-1}$ below and $108.1 \mathrm{~cm}^{-1}$ above the dissociation limit of the $\mathrm{Ar}-\mathrm{Br}$ potential, respectively, while in the case of $\mathrm{Ar}-\mathrm{Cl}$ these energy levels are $104.0 \mathrm{~cm}^{-1}$ below and $99.8 \mathrm{~cm}^{-1}$ above the dissociation limit, respectively. This means that $A r-B r$ can also accommodate some more available energy as internal energy than $\mathrm{Ar}-\mathrm{Cl}$. From the above energy considerations the gentle recoil mechanism would predict that the survival probability of $A r-B r$ should be higher than that of $\mathrm{Ar}-\mathrm{Cl}$. The second factor is related to sterical aspects of the initial state. The angular distribution of the $A r-H B r(v=1)$ ground vdW state, although similar to that of $A r-H C l(v=0)$, spreads more towards larger angles. The maximum of the $\operatorname{Ar}-\operatorname{HCl}(v=0)$ distribution occurs at $\theta=30.8^{\circ}$, while in the case of $A r-H B r(v=1)$ is at $\theta=33.6^{\circ}$, and the latter distribution has a higher probability at cluster geometries where the $H$ fragment is unblocked. In addition, the $A r-B r$ equilibrium separation $(\sim 4.3 \AA)$ in the initial state is slightly larger than that of $A r-C l(\sim 4.0 \AA)$. This more unblocked initial situation of the hydrogen in $A r-H B r(v=1)$ reduces the probability of collisions between the recoiling $H$ and the $A r$ atom, and would contribute to enhance the probability of $\mathrm{Ar}-\mathrm{Br}$ formation with respect to the $\mathrm{Ar}-\mathrm{Cl}$ case.

The effect of the shape of the initial distribution of intracluster orientations appears clearly in the $P_{A r-B r}(E) / P_{t o t a l}(E)$ ratio corresponding to the first excited vdW state of $A r-H B r(v=1)$ when compared to that of the ground vdW state. 
Since the shape of the $R$ dependence of $\psi(R, \theta)$ [Eq. (4)] is very similar for both vdW states, we conclude that the differences found in the yield of $A r-B r$ radicals are due to the different shape of the initial angular distributions. The yield of $A r-B r$ radical products for the excited vdW state of the cluster ranges from $80 \%$ for the lowest excitation energies to as high as $20 \%$ for the highest cluster energies investigated. The percentage of radical formation from the excited vdW state is larger than that from the ground vdW state by a factor $1.2-1.5$ for low and medium excitation energies, and by a factor 1.8 for very high energies. The present results therefore show a pronounced effect of the shape of the initial distribution of intracluster hydrogen orientations on the yield of the different fragments produced upon cluster photolysis.

It should be noted that quantum mechanical simulations of the $\mathrm{Ar}-\mathrm{HCl}(v=0)$ photolysis predicted a lower yield of $\mathrm{Ar}-\mathrm{Cl}$ radicals than the classical simulations, ${ }^{19}$ due to quantum effects such as interference between the PF and TF pathways. The origin of this interference between fragmentation pathways was attributed to the first collision of the recoiling $H$ fragment with the $A r$ atom. ${ }^{19}$ Thus, in a quantum mechanical description of the $A r-H B r(v=1)$ photolysis the predicted yield of $A r-B r$ radicals could change to some extent with respect to the classical ones of Fig. 2. However, since the interference effects are caused by the first $H / A r$ collision, it is expected that they will affect less the photolysis process in $A r-\operatorname{HBr}(v=1)$ than in $A r-H C l(v=0)$. Indeed, the higher classical probability of $A r-B r$ radicals for the two vdW states of $A r-H B r(v=1)$, as compared with the classical yield of $\mathrm{Ar}-\mathrm{Cl}$ products, indicates that $H / A r$ collisions occur with much lower probability in $A r-H B r(v=1)$ than in $A r-H C l(v=0)$. In particular, photolysis from the excited vdW state of $A r-\operatorname{HBr}(v=1)$ is expected to be less affected by interference effects than from the ground vdW one, due to its initial angular geometry distribution, for which the recoiling hydrogen is more likely to miss the $A r$ atom. Thus, if interference effects are relevant in the $A r-H B r(v=1)$ photolysis and they act to diminish the classical yield of radicals [as in $\mathrm{Ar}-H \mathrm{Cl}(v=0)]$, the 
differences between the yield of radicals obtained from the ground and the excited vdW states could be even larger than those found in Fig. 2.

An interesting question now is how the effect of the shape of the initial angular distribution manifests in the state distributions of the fragments produced by the $\mathrm{PF}$ and TF pathways. This is analyzed in the remaining of this Section.

\section{C. $A r-B r$ product state distributions}

Rovibrational state distributions of the $A r-B r$ fragment were calculated, and are shown in Fig. 3 for the initial ground and first excited vdW state of $A r-\operatorname{HBr}(v=$ 1 ), respectively, for different cluster excitation energies. The negative $E_{\nu, j}$ energies correspond to the bound states of $A r-B r$, while the positive energies correspond to the quasibound states. The discrete distributions of Fig. 3 are obtained by discretizing the classical angular momentum $p_{\theta^{\prime}}$ and energy $E_{A r-B r}^{c l a s s}$. As commented above, the rotational state $j$ corresponding to each trajectory is identified by solving the equation $p_{\theta^{\prime}}^{2}=j(j+1)$. Then each trajectory leading to an $A r-B r$ product is assigned a vibrational state $\nu$ and a rovibrational energy level $E_{\nu, j}$, by identifying the quantum energy level $E_{\nu, j}$ [obtained from Eq. (8)] which is closest to the classical energy $E_{A r-B r}^{c l a s s}$ of the trajectory. The distributions of Fig. 3 can be obtained experimentally either from the time-of-flight (TOF) spectrum of the hydrogen fragment, due to conservation of the total energy of the system, or by probing directly the $A r-B r$ product.

The effect of increasing excitation energy in the rovibrational distributions of both vdW states is that they gradually shift towards higher bound and quasibound states of $A r-B r$. This is caused by the increase of the energy available for the radical. It is interesting to observe that even at high excitation energies (like $E=$ $5.25 \mathrm{eV}$ ) the maximum population of the distributions still do not reach the highest quasibound states of $\mathrm{Ar}-\mathrm{Br}$. In the case of $\mathrm{Ar}-\mathrm{HCl}(v=0)$ photolysis, already for excitation energies $E \simeq 2 e V$ the $A r-C l$ rovibrational distributions peaked at the highest quasibound states of the radical fragment. ${ }^{19}$ This is a result of the smaller 
share of energy available for $\mathrm{Ar}-\mathrm{Br}$ with respect to $\mathrm{Ar}-\mathrm{Cl}$ discussed above.

For the same cluster excitation energy, the rovibrational distributions produced by $A r-H B r(v=1)$ photolysis from the ground and first excited vdW states exhibit a different shape. The main difference is that the $A r-B r$ fragments produced from the ground vdW state are hotter than those produced from the first excited vdW state. The distributions of the excited vdW state present a substantially higher population in the $A r-B r$ bound states, particularly the low and medium ones. In order to analyze in more detail the differences in the shape of the distributions of Fig. 3, vibrational and rotational distributions have been calculated, and are presented in Figs. 4 and 5, respectively.

The vibrational distributions obtained for the two vdW states of $A r-H B r(v=$ 1) are cold and rather similar. Some more vibrational excitation is found in general in the distributions associated with the excited vdW state. With increasing excitation energy the vibrational distribution of the excited vdW state does not change much, while that of the ground vdW state becomes gradually colder, increasing the differences between the two vibrational distributions. More pronounced differences are found between the $j$-state distributions in Fig. 5. The distributions associated with the ground vdW state are clearly hotter than the ones corresponding to the excited vdW state. The $j$ distributions of the ground vdW state peak at the highest $j$ states populated, with a tail of decreasing intensity extending towards lower $j$ values, while in the case of the excited vdW state the population distributes rather uniformly in the range of populated $j$ states.

The following picture of the photodissociation dynamics leading to $\mathrm{Ar}-\mathrm{Br}$ radicals emerges from the above state distributions. The $A r-B r$ fragments are produced upon direct dissociation of the hydrogen. For initial orientations of the hydrogen corresponding to the distribution of the ground vdW state of $A r-\operatorname{HBr}(v=1)$, mostly in the range $\theta<80^{\circ}$ (see Fig. 1), the recoiling $H$ produces a large torque on the $\mathrm{Ar}-\mathrm{Br}$ fragment. As a consequence of this large torque, the excitation of $A r-B r$ goes mainly to rotation of the fragment, consistently with the highly ex- 
cited rotational distributions of Fig. 5 and the cold vibrational distributions of Fig. 4. By contrast, when the hydrogen recoils from the initial orientations determined by the excited vdW state, it produces a smaller torque on the radical fragment, leading to substantially less rotational excitation. For this distribution of initial orientations, mainly in the region of the $A r-B r-H$ collinear geometry, vibrational excitation of the $A r-B r$ product is more favored than for the ground vdW state initial distribution, consistently with the results of Fig. 4.

The result that the rovibrational distributions of the ground vdW state are energetically more excited than the corresponding ones of the excited vdW state can be understood in terms of the higher rotational excitation produced by photolysis from the ground vdW state. Most of the vibrational population of $A r-B r$ is concentrated in the same states, $\nu=0,1,2,3$, for both initial vdW states of the cluster. The energy location of these vibrational states becomes increasingly higher as $j$ increases. Therefore, the higher rotational excitation produced from the ground vdW state is the origin of the higher energetical excitation of the corresponding rovibrational distributions, as compared with those of the excited vdW state. Likewise, the different shape of the distributions associated with the two vdW states is mainly due to the differences in the corresponding rotational distributions.

The average $j$-state distributions of Fig. 5 reflect the shape of the rotational progressions of the $A r-B r$ vibrational states, and particularly of the most populated ones. Inspection of the quantum mechanical energies $E_{\nu, j}$ shows that for low $j$ states the separation between the lowest vibrational levels $\nu=0,1,2,3$ ranges between 27 $\mathrm{cm}^{-1}$ and $19 \mathrm{~cm}^{-1}$. Taking into account the above energy separation and the shape of the $j$ distributions of Fig. 5, the progressions corresponding to the $\nu=0,1$ states can be clearly identified in general in the distributions of Fig. 3. In the left panels of Fig. 3, for $E=1.83 \mathrm{eV}$ and $E=2.97 \mathrm{eV}$ the maxima of the $\nu=0,1$ progressions (and also of the $\nu=2$ progression for $E=1.83 \mathrm{eV}$ ) are well separated in energy. In the case of $E=2.97 \mathrm{eV}$ even the $\nu=2,3$ progressions can be distinguished in the excited vdW state distribution. For high energies like $E=5.25 \mathrm{eV}$ the $\nu=0$ 
progression, with the maximum population at very high quasibound states, clearly differenciates from the other $\nu>0$ progressions, for both vdW states. Thus, with enough resolution the whole discrete spectrum of the radical can be probed from the rovibrational distributions produced after UV photolysis of the cluster at different excitation energies.

\section{D. $H$ fragment distributions}

The influence of the shape of the initial angular distribution of the cluster in the final $H$ fragment distributions has also been investigated. Kinetic-energy distributions of $\mathrm{H}$ produced through the TF pathway, and angular distributions of the $H$ product obtained both by partial and total fragmentation have been calculated for the two initial vdW states of the cluster, and are shown in Figs. 6 and 7, respectively.

The hydrogen kinetic-energy distributions of Fig. 6 are the equivalent, in the energy domain, to the $H$ fragment TOF spectrum. The distributions consist of a peak at hydrogen kinetic energies, $\epsilon_{k}^{H}$, slightly lower than the cluster excitation energy $E$, and a tail extending towards lower kinetic energies. The peak at the highest $\epsilon_{k}^{H}$ values of the distributions is produced by nearly direct dissociation of the hydrogen which, upon recoiling, collides weakly with the $A r$ obstacle. In this weak, side $H / A r$ collision the hydrogen transfers a small amount of energy to the $A r-B r$ bond (albeit enough to break it), and the $H$ fragment escapes carrying most of the excess energy of the system. The tail at lower kinetic energies in the distributions is caused by one hard $H / A r$ collision or several successive (weak and hard) $H / A r$ and $H / B r$ collisions. Depending on the number and the strength of the collisions, the initially hot $H$ fragment is more or less effectively cooled down. The shape of the hydrogen kinetic-energy distribution (KED) is qualitatively similar for the different excitation energies. The main effect of increasing $E$ in the $H$ KED is that the distribution tail reaches lower $\epsilon_{k}^{H}$ values, since the hotter hydrogen transfers more energy in each collision.

When the hydrogen distributions obtained from the two initial vdW states of 
the cluster are compared for the same excitation energy, it is observed that the low kinetic-energy tail is substantially less intense in the distributions of the excited vdW state (for all energies $E$ ). Practically all the population goes to the high kinetic-energy peak of the distributions. This result is again due to the shape of the initial distribution of hydrogen orientations. In the case of the first excited vdW state of $A r-H B r(v=1)$, the initial angular distribution favors direct escaping of the hydrogen and a much smaller probability of $H / A r$ and $H / B r$ collisions than for the ground vdW state. The low kinetic-energy tail in the $H$ fragment KED is the signature of caging of the hydrogen within a cluster with a single $A r$ solvent atom. In this sense, the present effect of a diminishing tail is equivalent to that found in larger $A r_{n}-H X$ clusters, ${ }^{22,25(b), 27}$ i.e., that the caging of the hydrogen is "turned off" for initial angular distributions where the hydrogen recoil is not hindered.

In the $H$ product KED obtained when the TOF spectrum is transformed to the kinetic-energy domain there is population corresponding to $H$ fragments produced via both the PF and the TF pathways. For the sake of clarity, these two contributions have been separated in this work into the discrete rovibrational distributions of Fig. 3, and the continuum kinetic-energy distributions of Fig. 6. When the two contributions appear together in a single distribution there is a region of kinetic energies where they overlap. This region corresponds to the region of the radical quasibound states, $E_{\nu, j}>0$ in the rovibrational distributions of Fig. 3, and the region of highest $\epsilon_{k}^{H}$ energies of the peak in the TF distributions of Fig. 6. In the regions of the whole $H$ KED corresponding to the low kinetic-energy tail and the radical bound states, $E_{\nu, j}<0$, the TF and PF populations are well separated.

The present results of Figs. 3 and 6 predict that the effect of the shape of the initial distribution of hydrogen intracluster orientations manifests clearly at least in the above two kinetic energy regions of the $H$ fragment distribution. Indeed, for initial angular distributions where the hydrogen recoil becomes less hindered, the intensity of the red-shifted tail decreases and the blue-shifted population associated with radical bound states increases. Such manifestations are experimentally observ- 
able, and can be used to determine the degree of initial caging of the hydrogen for the cluster state prepared in the excited electronic surface.

The final hydrogen angular distributions corresponding to each vdW state and to each fragmentation pathway exhibit a similar shape for the different cluster excitation energies, and only distributions for two excitation energies are shown in Fig. 7. For the same initial vdW state the main difference between the PF and TF angular distributions is that in the latter distributions a peak of intensity appears in the angular region $100^{\circ} \leq \theta^{\prime} \leq 130^{\circ}$. In addition, in the case of the excited vdW state the peak around $\theta^{\prime} \simeq 40^{\circ}$ is more intense in the TF angular distributions than in the PF ones.

The peaks around $\theta^{\prime} \simeq 40^{\circ}$ and $\theta^{\prime} \simeq 120^{\circ}$ in the TF distributions of both vdW states correspond to $H$ fragments scattered after one or more collisions with $A r$ and $\mathrm{Br}$, respectively. These peaks are originated from initial hydrogen orientations typically $\theta<80^{\circ}$ (see Fig. 1). In all the distributions of Fig.7, the region $\theta^{\prime}<$ $30^{\circ}-35^{\circ}$ with no population is due to the angular size of the Ar obstacle, while the intensity appearing at $\theta^{\prime}>130^{\circ}$ (coming from initial orientations $\theta>130^{\circ}$ ) is produced by practically direct $H$ recoiling, with weak $H-A r$ interaction. In this latter case, when TF is produced, the energy available for $A r-B r$ is enough to break this bond. The intensity of the peaks around $\theta^{\prime} \simeq 40^{\circ}$ and $\theta^{\prime} \simeq 120^{\circ}$ is significantly smaller in the TF angular distributions of the excited vdW state, in comparison with those of the ground vdW state. This is consistent with the less intense tails of the distributions shown in the lower panels of Fig. 6, associated with a lower probability of several collisions between the hydrogen and the heavier atoms.

Interestingly, the angular distributions associated to $H$ fragments produced by both the PF and the TF pathways reflect the shape of the initial angular distribution from which they are originated. This result is not surprising, since in both the $\mathrm{PF}$ and the TF pathways the dominant mechanism is direct or nearly direct dissociation of the hydrogen. Some additional structure is introduced in the TF angular distributions by indirect dissociation dynamics, which is reflected in the peak 
around $\theta^{\prime} \simeq 120^{\circ}$ due to escaping of the hydrogen after bouncing once or more in between $\mathrm{Ar}$ and $\mathrm{Br}$. Therefore, the angular distribution of the $H$ photofragments could be used as a probe of the initial distribution of intracluster hydrogen orientations excited to the upper electronic surface. This would provide a means to map the shape of the angular wave function of the cluster states in the lower electronic surface, prepared by infrared excitation prior to the UV pumping. It should be noted, however, that the angular distributions of Fig. 7 are calculated in the cluster body-fixed frame, and are not directly comparable with experimental angular distributions measured in the laboratory frame. The angular distributions measured in the laboratory frame might not reflect the angular shape of the initial state as clearly as the present body-fixed frame distributions do.

\section{CONCLUDING REMARKS}

The ultraviolet photolysis of the $A r-H B r$ cluster is studied by means of a quasiclassical trajectory method. The main goal is to investigate how the shape of the initial distribution of intracluster angular geometries affects the yield of the different photoproducts and their state distributions. To this purpose the photolysis of $\operatorname{Ar}-\operatorname{HBr}(v=1)$ is simulated starting from two different initial states of the cluster. One of them is the ground vdW state of $A r-\operatorname{HBr}(v=1)$, for which the equilibrium geometry corresponds to the $\mathrm{Ar}-\mathrm{H}-\mathrm{Br}$ isomer, where hydrogen dissociation is initially hindered with a high probability. The other state is the first excited vdW state of $A r-H B r(v=1)$, associated with the equilibrium geometry of the $\mathrm{Ar}-\mathrm{Br}-\mathrm{H}$ isomer. In this case hydrogen dissociation is not hindered for the most likely initial orientations. Simulations are carried out for different excitation energies of the cluster in the range of the absorption spectrum.

Cluster photolysis occurs through two different fragmentation pathways, namely partial fragmentation into $H+A r-B r$ radicals, and total fragmentation into $H+$ $A r+B r$. The yield of $A r-B r$ products is found to be high for the two initial states of the cluster, in contrast with the low yield of $\mathrm{Ar}-\mathrm{Cl}$ radicals previously 
found in the case of $\mathrm{Ar}-\mathrm{HCl}$. The yield of $\mathrm{Ar}-\mathrm{Br}$ radicals produced from the first excited vdW state is remarkably higher, by a factor ranging between 1.2 and 1.8, depending on the excitation energy, with respect to the yield obtained from the ground vdW state. This higher yield is attributed to the initial distribution of intracluster orientations of the excited vdW state, from which the $H$ fragment can recoil directly leaving behind $A r-B r$ radicals, with a higher probability than in the case of the ground vdW state.

Manifestations of the effect of the initial angular distribution shape are found as well in the state distributions of the different photolysis products. Specifically, in the case of the excited vdW state of the cluster, the $A r-B r$ products exhibit less rovibrational excitation, and the kinetic-energy distributions of the $H$ fragments produced by total fragmentation are hotter. Such differences are the result of a photodissociation dynamics influenced to a large extent by the specific shape of the distribution of initial hydrogen intracluster orientations. When the hydrogen is less caged in between $\mathrm{Ar}$ and $\mathrm{Br}$ the photodissociation process becomes more direct, decreasing the probability of energy transfer between the recoiling $H$ and the $A r-B r$ subunit. The present simulations show that the final angular distributions of the $H$ fragment (produced either by the partial or the total fragmentation pathway) reflects closely the shape of the initial angular distribution of the cluster.

Selecting the cluster initial state based on the shape of its angular distribution appears as an efficient means to control the yield of the different photolysis products and the states in which they are produced. In this work two initial states with rather opposite angular shapes are investigated. Other cluster states with intermediate angular shapes are likely to be found, which would allow for a higher degree of control. Finally, the trends found here are nor limited to $A r-H B r$, and they appear to be general for other related hydrogen-bonded clusters. Experimental investigation of such trends would be very interesting.

\section{ACKNOWLEDGMENTS}


This work was supported by C.I.C.Y.T., Spain, Grant No. BCM-2001-2179, and by the European network TMR, Grant No. HPRN-CT-1999-00005.

\section{References}

[1] J. Segall, Y. Wen, R. Singer, C. Wittig, A. García-Vela, and R.B. Gerber, Chem. Phys. Lett. 207, 504 (1993).

[2] C. Jaques, L. Valachovic, S. Ionov, Y. Wen, E. Böhmer, J. Segall, and C. Wittig, J. Chem. Soc. Faraday Trans. 89, 1419 (1993).

[3] J. Zhang, M. Dulligan, J. Segall, Y. Wen, and C. Wittig, J. Phys. Chem. 99, 13680 (1995).

[4] K. Liu, A. Kolessov, J.W. Partin, I. Bezel, and C. Wittig, Chem. Phys. Lett, 299, 374 (1999).

[5] C.A. Picconatto, H. Ni, A. Srivastava, and J.J. Valentini, J. Chem. Phys. 114, 7073 (2001).

[6] D.F. Plusquellic, O. Votava, and D.J. Nesbitt, J. Chem. Phys. 101, 6356 (1994).

[7] O. Votava, D.F. Plusquellic, T.L. Myers, and D.J. Nesbitt, J. Chem. Phys. 112, 7449 (2000).

[8] S.R. Mackenzie, O. Votava, J.R. Fair, and D.J. Nesbitt, J. Chem. Phys. 105, 11360 (1996); 110, 5149 (1999).

[9] M.A. Young, J. Chem. Phys. 102, 7925 (1995).

[10] R. Baumfalk, U. Buck, C. Frischkorn, N.H. Nahler, and L. Hüwel, J. Chem. Phys. 111, 2595 (1999).

[11] R. Alimi and R.B. Gerber, Phys. Rev. Lett. 64, 1453 (1990). 
[12] A. García-Vela, R.B. Gerber, and J.J. Valentini, Chem. Phys. Lett. 186, 223 (1991); J. Chem. Phys. 97, 3297 (1992).

[13] A. García-Vela, R.B. Gerber, D.G. Imre, and J.J. Valentini, Phys. Rev. Lett. 71, 931 (1993).

[14] A. García-Vela and R.B. Gerber, J. Chem. Phys. 98, 427 (1993).

[15] T. Schröder, R. Schinke, M. Mandziuk, and Z. Bačić, J. Chem. Phys. 100, 7239 (1994); T. Schröder, R. Schinke, and Z. Bačić, Chem. Phys. Lett. 235, $316(1995)$.

[16] A. García-Vela, J. Chem. Phys. 108, 5755 (1998).

[17] E. Narevicius and N. Moiseyev, Chem. Phys. Lett. 287, 250 (1998); Mol. Phys. 94, 897 (1998).

[18] J.C. Juanes-Marcos and A. García-Vela, J. Chem. Phys. 112, 4983 (2000); 115, $5692(2001)(\mathrm{E})$.

[19] J.C. Juanes-Marcos and A. García-Vela, J. Phys. Chem. A 106, 236 (2002).

[20] A. García-Vela, R.B. Gerber, and U. Buck, J. Phys. Chem. 98, 3518 (1994).

[21] M.Y. Niv, A.I. Krylov, and R.B. Gerber, Faraday Discuss. 108, 243 (1997); M.Y. Niv, A.I. Krylov, R.B. Gerber, and U. Buck, J. Chem. Phys. 110, 11047 (1999).

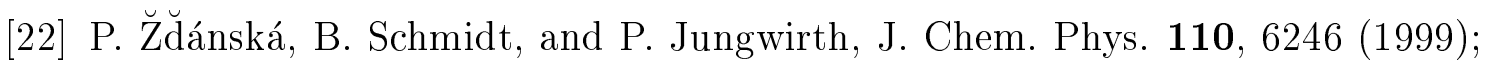

P. Z̆ đánská, P. Slavíček, and P. Jungwirth, ibid. 112, 10761 (2000).

[23] A.B. McCoy, Y. Hurwitz, and R.B. Gerber, J. Phys. Chem. A 97, 12516 (1993).

[24] M. Monnerville and B. Pouilly, Chem. Phys. Lett. 294, 473 (1998). 
[25] (a) R. Baumfalk, N.H. Nahler, U. Buck, M.Y. Niv, and R.B. Gerber, J. Chem. Phys. 113, 329 (2000); (b) P. Slavíček, P. Z̆ ̌̆ánská, P. Jungwirth, R. Baumfalk, and U. Buck, J. Phys. Chem. A 104, 7793 (2000).

[26] T. Schröder, R. Schinke, S. Liu, Z. Bačić, and J.W. Moskowitz, J. Chem. Phys. 103, 9228 (1995).

[27] B. Schmidt, Chem. Phys. Lett. 301, 207 (1999).

[28] K.M. Christoffel and J.M. Bowman, J. Chem. Phys. 104, 8348 (1996).

[29] J.R. Fair, and D.J. Nesbitt, J. Chem. Phys. 113, 10962 (2000).

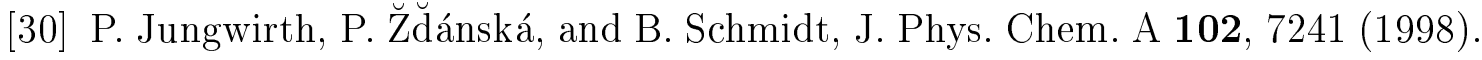

[31] J.M. Hutson, J. Chem. Phys. 91, 4455 (1989).

[32] A.L. McIntosh, Z. Wang, J. Castillo-Chará, R.R. Lucchese, J.W. Bevan, R.D. Suenram, and A.C. Legon, J. Chem. Phys. 111, 5764 (1999).

[33] J. Han, A.L. McIntosh, Z. Wang, R.R. Lucchese, and J.W. Bevan, Chem. Phys. Lett. 265, 209 (1997).

[34] G. Herzberg, Molecular Spectra and Molecular Structure (Krieger, Malabar, 1989), Vol. 1.

[35] D.A. Chapman, K. Balasubramanian, and S.H. Lin, Chem. Phys. 118, 333 (1987).

[36] Z.-H. Huang and H. Guo, J. Chem. Phys. 96, 8564 (1992).

[37] K.T. Tang and J.P. Toennies, Chem. Phys. 156, 413 (1991).

[38] P. Casavecchia, G. He, R.K. Sparks, and Y.T. Lee, J. Chem. Phys. 75, 710 (1981).

[39] A. García-Vela, J. Chem. Phys. 112, 8302 (2000). 


\section{FIGURE CAPTIONS}

Fig. 1. Angular distributions of the initial $A r-H B r(v=1)$ ground (solid line) and first excited (dashed line) vdW state calculated through Eq. (5). The initial angular distribution of the $\mathrm{Ar}-\mathrm{HCl}(v=0)$ ground vdW state (dotted line) is also shown for comparison. All the distributions are normalized to unity.

Fig. 2. Ratio between the probability of $A r-X$ formation, $P_{A r-X}(X=B r, C l)$, and the total probability of photodissociation, $P_{\text {total }}$ vs $E$. The ratios calculated for the initial ground and first excited vdW states of $A r-H B r(v=1)$ are shown and compared with the ratio previously obtained (Ref. 19) for the ground vdW state of $A r-H C l(v=0)$. The limit $E=0$ corresponds to three separated atoms.

Fig. 3. Rovibrational state distributions of the $A r-B r$ radical fragment for three different excitation energies, when photolysis is started from the ground (left panels) and the first excited (right panels) vdW state of $A r-H B r(v=1)$. All the distributions are normalized to unity.

Fig. 4. Vibrational distributions of the $A r-B r$ product for four different excitation energies of the parent cluster, when photolysis starts from the ground (solid lines) and first excited (dashed lines) vdW state of $A r-H B r(v=1)$. All the distributions are normalized to unity.

Fig. 5. Rotational distributions of $A r-B r$ for three excitation energies, starting from the initial ground and first excited vdW state of $A r-H B r(v=1)$. All the distributions are normalized to unity.

Fig. 6. Kinetic-energy distributions of the $H$ product of the total fragmentation pathway for three excitation energies of $\operatorname{Ar}-\operatorname{HBr}(v=1)$ after photolysis initiated from the ground and first excited vdW state of the cluster. All the distributions are normalized to unity. 
Fig. 7. Angular distributions of the $H$ fragment produced by the partial fragmentation (four upper panels) and the total fragmentation (four lower panels) pathway for two cluster excitation energies, obtained after photolysis from the initial ground and first excited state of $A r-H B r(v=1)$. All the distributions are normalized to unity. 